\title{
Clinical Evaluation of Hearing Loss and Tinnitus in Secretory Otitis Media
}

\author{
Ying Gao, Qing Zhang, Huanan Luo, Botao Wang* \\ Department of Otolaryngology, The Second Affiliated Hospital of Xi'an Jiaotong University, Xi'an, China \\ Email: *zhqent@163.com
}

How to cite this paper: Gao, Y., Zhang, Q., Luo, H.N. and Wang, B.T. (2018) Clinical Evaluation of Hearing Loss and Tinnitus in Secretory Otitis Media. Journal of Biosciences and Medicines, 6, 50-57. https://doi.org/10.4236/jbm.2018.611006

Received: October 23, 2018

Accepted: November 13, 2018

Published: November 16, 2018

Copyright $\odot 2018$ by authors and Scientific Research Publishing Inc. This work is licensed under the Creative Commons Attribution International License (CC BY 4.0).

http://creativecommons.org/licenses/by/4.0/ (c) (i) Open Access

\begin{abstract}
Background: Secretory otitis media (SOM) patients usually complain about tinnitus accompanied with a sensation of hearing loss and ear fullness. Investigation of the association between Sensorineural hearing loss (SNHL) and tinnitus has been rarely reported. Methods: The clinical records of 34 patients with unilateral SOM accompanied with tinnitus were reviewed in search of bone conduct (BC) hearing thresholds and Tinnitus Handicap Inventory (THI). All the enrolled patients take audiogram and THI preoperative and three month's postoperative between February 2015 and January 2016. Differences between the affected side and the other in BC threshold, preoperatively and postoperatively were calculated. Correlations between $\mathrm{BC}$ thresholds' lose and change of the THI scores' postoperative data were analyzed. Results: The mean $\mathrm{BC}$ thresholds of the SOM ears preoperative were $18.3 \pm 10.7 \mathrm{dBHL}$, which was higher than the other ear $(16.6 \pm 8.8 \mathrm{dBHL}, t=$ $2.105, \mathrm{p}<0.05$ ), especially in $2 \mathrm{kHz}$ frequency of pure-tone audiometry (Affected side: $24.7 \pm 13.7 \mathrm{dBHL}$, Non-affected side: $20.4 \pm 10.5 \mathrm{dBHL}, t=3.510$, $\mathrm{p}<0.01)$. There exists a statistically significant difference in the mean THI scores $(t=6.958, \mathrm{p}<0.01)$ between pre $(23.5 \pm 6.9)$ and postoperatively (16.0 \pm 5.5 ), so does in the BC thresholds (pre: $17.0 \pm 8.1 \mathrm{dBHL}$, post: $16.2 \pm 7.9$ $\mathrm{dBHL}, t=3.195, \mathrm{p}<0.01$ ) and $2 \mathrm{kHz}$ frequency (pre: $23.4 \pm 11.7 \mathrm{dBHL}$, post: $21.3 \pm 10.4 \mathrm{dBHL}, t=3.057, \mathrm{p}<0.01)$. In addition, there is correlation between THI scores and average BC thresholds $(r=0.37, p=0.03)$, THI scores and $2 \mathrm{kHz}(\mathrm{r}=0.42, \mathrm{p}=0.01)$ as well as THI scores and Air-bone gap (ABG) frequency $(r=0.60, p<0.01)$ in the patients. Conclusion: SOM could bring about SNHL and tinnitus which reduced postoperatively. In addition, Tinnitus has correlation with bone-conduct hearing thresholds and conductive hearing loss.
\end{abstract}

\section{Keywords}

Sensorineural Hearing Loss, Tinnitus, Secretory Otitis Media 


\section{Background}

SOM can be defined as the presence of middle ear effusion (serous, purulent, mucoid, or a mixture of these) in the absence of acute ear infection [1]. Persistent middle-ear fluid caused by SOM results in decreased mobility of the tympanic membrane and dysfunctional sound conduction [2]. The SNHL is commonly observed in chronic otitis media (COM) patients confirmed by the evidence that much more severe pathological changes appeared in the basal turns of the cochlea [3]. In addition, the studies also found that otitis media related SNHL accounts for $1 \%-9 \%$ of cases [4] [5] [6] [7]. Clinically, we found the same thing which came across in the SOM patients. Some SOM patients usually complain about tinnitus accompanied with a sensation of hearing loss and ear fullness, which could not be totally cured and eliminated after receiving the therapy of myringotomy with or without tube insertion [6].

The mechanism of tinnitus is involved in the auditory input to the auditory nervous system. As the major kinds of abnormalities resulted from the loss of input and neural plasticity, hyperactivity auditory pathway, re-organization (re-mapping) of neural structures and re-routing of sensory information [8] may make the contribution to the development of severe tinnitus. As the hearings of patients with SOM could be restored after surgery, we hypothesized that the tinnitus symptoms could be postoperatively alleviated. Therefore, the present research was carried out to evaluate the potential changes about tinnitus as well as analyze the correlation between tinnitus and hearing loss.

\section{Methods}

\subsection{Material}

The proposed study was conducted in patients with unilateral secretory otitis media accompanied with tinnitus who received myringotomy or inserting ventilation tube between February 2015 and January 2016 in the Second Affiliated Hospital of Xi'an Jiaotong University, Shaanxi, China, and all of them were performed by one senior surgeon. Among this cohort, patients with tinnitus just in the non-operative sides or with unfinished data in their preoperative medical records were not involved. Inclusion criteria were as follows: 1) SOM is limited to just one side rather than both sides; 2) Tinnitus is lateralized on the SOM affected side or non-lateralized behind the onset of SOM. Exclusive criteria were: 1) with teeth orcraniocervical muscular diseases; 2) with nasopharyngeal carcinoma , acoustic neuroma, brain-stem tumor, infarction and multiple sclerosis; 3) possessing a history of sensorineural hearing loss, suppurative otitis media, otosclerosis, Meniere's disease and vertigo history; 4) with a history of special drug therapy such as aspirin and nonsteroidal anti-inflammatory drugs, aminoglycosides and vancomycin as well as chemotherapeutics and loop diuretics; 5) with metabolic and psychogenic disease. All the patients were arranged for physical and ENT special examinations, laryngoscopy and temporal high-resolution CT scan preoperatively. Audiologic evaluation by pure tone audiometry was carried 
out prior to and 3 months after the surgery. Bone-conduction hearing levels at $0.25,0.5,1,2$ and $4 \mathrm{kHz}$ were recorded and the average bone-conduction threshold was calculated according to $0.25,0.5,1,2 \mathrm{kHz}$ frequency. In the meanwhile, THI scores were also recorded to investigate the potential changes about tinnitus.

\subsection{Statistical Analysis}

The analysis of all the data was made with the application of SPSS software (ver. 16.0), and p-value less than 0.05 was considered to have statistical significance. In particular, paired $t$ test was not only used for the comparison of the $\mathrm{BC}$ threshold and tinnitus/THI scores between the affected ear and non-affected ear, but also for the affected ear preoperatively and postoperatively. At the same time, Pearson Correlation Coefficient was used to evaluate the correlation among average $\mathrm{BC}$ threshold, $\mathrm{ABG}$ and tinnitus/THI scores.

\section{Results}

\subsection{Clinical Characteristics of Patients with SOM}

The mean age of the SOM patients enrolled in the proposed study (34 cases) was $43.8 \pm 16.5$ years old, and the SOM history lasted for $3-14$ months (mean 8 months). 6 cases had been treated with myringotomy during the earlier therapy. Among them, 13 cases reported that their tinnitus symptoms appeared in the affected ears and the rest (21 cases) stated tinnitus from both sides of the ear or the head. Table 1 showed the characteristics of all the patients.

\subsection{Analysis of BC Frequencies between Affected and Non-Affected Side Preoperatively}

Overall, the average BC threshold of the affected side for SOM patients (18.3 \pm $10.7 \mathrm{dBHL})$ was obviously higher than that of the non-affected side $(16.6 \pm 8.8$ $\mathrm{dBHL})(t=3.18, \mathrm{p}=0.04)$. However, when the BC threshold in each frequency was compared between the affected side and non-affected side one by one, significant difference could just be seen in $2 \mathrm{kHz}(t=3.51, \mathrm{p}=0.001)$, as shown in Figure 1 (a) and Table 2.

\subsection{Analysis of THI Score, BC Hearing Frequencies between Pre and Postoperative of SOM Affected Side}

Although five cases were lost to follow-up, the mean THI score of the remaining patients after operation was $16.3 \pm 5.2$, which was obviously lower than that of patients prior to the surgery $(23.9 \pm 6.4)(t=7.21, \mathrm{p}=0.00)$. In the meanwhile, the mean value of $\mathrm{BC}$ average threshold for the preoperative patients in the affected side $(16.8 \pm 8.0 \mathrm{dBHL})$ was remarkably lower than that for the postoperative patients $(16.0 \pm 7.8 \mathrm{dBHL})(t=3.18, \mathrm{p}=0.004)$. Furthermore, according to Table 3 and Figure 1(b), significant differences concerning preoperative and postoperative $\mathrm{BC}$ hearing levels could be found in all frequencies except for 0.25 


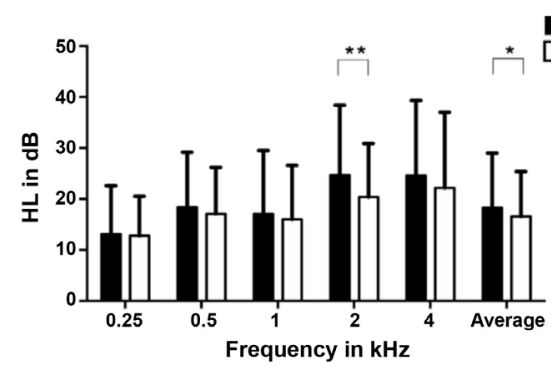

(a)

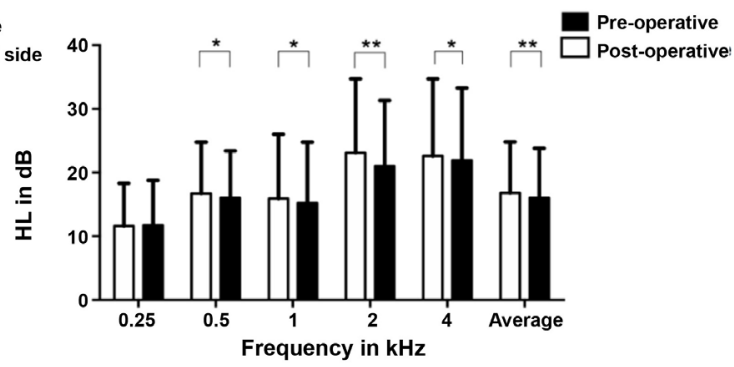

(b)

Figure 1. Analysis of BC frequencies (a) between affected and non-affected side preoperatively; (b) between pre and postoperative of OME affected side.

Table 1. Patient Characteristics. ${ }^{*} \mathrm{p}<0.05,{ }^{* *} \mathrm{p}<0.01$.

\begin{tabular}{cc}
\hline Variable & All Patients $(\mathrm{N}=34)$ \\
\hline Age, $\mathrm{y}($ range $)$ & $43(11-66)$ \\
Gender, n (\%) & $17(50)$ \\
Male & $17(50)$ \\
Female & \\
Affected side, $\mathrm{n}(\%)$ & $19(56)$ \\
Right & $15(44)$ \\
Left & \\
B & $23(68)$ \\
C & $11(32)$ \\
Acousticimmittance, $\mathrm{n}(\%)$ & $11(32)$ \\
Type of surgery, $\mathrm{n}(\%)$ & $23(68)$ \\
Tympanostomy &
\end{tabular}

Table 2. Analysis of BC frequencies between affected and non-affected side preoperatively.

\begin{tabular}{ccccccc}
\hline Frequency $(\mathrm{kHz})$ & 0.25 & 0.5 & 1 & 2 & 4 & $\begin{array}{c}\text { Average } \\
\text { threshold }\end{array}$ \\
\hline Affected side & $13.1 \pm 9.5$ & $18.4 \pm 10.8$ & $17.1 \pm 12.4$ & $24.7 \pm 13.7$ & $24.6 \pm 14.7$ & $18.3 \pm 10.7$ \\
Non-affected side & $12.8 \pm 7.7$ & $17.1 \pm 9.1$ & $16.0 \pm 10.6$ & $20.4 \pm 10.5^{\star *}$ & $22.2 \pm 14.8$ & $16.6 \pm 8.8^{*}$ \\
$t$ value & 0.33 & 1.22 & 0.93 & 3.51 & 1.85 & 3.18 \\
P value & 0.74 & 0.23 & 0.36 & 0.001 & 0.07 & 0.04
\end{tabular}

Compared to affected side: ${ }^{\star} \mathrm{p}<0.05,{ }^{* *} \mathrm{p}<0.01$.

Table 3. Analysis of BC frequencies between pre and postoperative of OME affected side.

\begin{tabular}{ccccccc}
\hline $\begin{array}{c}\text { Frequency } \\
(\mathrm{kHz})\end{array}$ & 0.25 & 0.5 & 1 & 2 & 4 & $\begin{array}{c}\text { Average } \\
\text { threshold }\end{array}$ \\
\hline Preoperative & $11.6 \pm 6.7$ & $16.7 \pm 8.1$ & $15.9 \pm 10.1$ & $23.1 \pm 11.6$ & $22.6 \pm 12.1$ & $16.8 \pm 8.0$ \\
Postoperative & $11.7 \pm 7.1$ & $16.0 \pm 7.4^{*}$ & $15.2 \pm 9.6^{*}$ & $21.0 \pm 10.3^{* *}$ & $21.9 \pm 11.4^{*}$ & $16.0 \pm 7.8^{* *}$ \\
$t$ value & 0.44 & 2.11 & 2.11 & 3.04 & 2.11 & 3.18 \\
P value & 0.66 & 0.04 & 0.04 & 0.005 & 0.04 & 0.004 \\
\hline
\end{tabular}

Compared to preoperative: ${ }^{\star} \mathrm{p}<0.05,{ }^{* *} \mathrm{p}<0.01$. 
$\mathrm{kHz}$, suggesting that the $\mathrm{BC}$ hearing levels could be improved for the SOM patients postoperatively.

\subsection{Correlation between THI Scores and BC Hearing Frequencies, THI Scores and ABG}

Our findings demonstrated that THI score given from SOM patients shows strong correlation with overall average $\mathrm{BC}$ threshold $(\mathrm{r}=0.37, \mathrm{p}=0.03)$ (Figure $2(\mathrm{a}))$ and the specific BC threshold in the frequency of $2 \mathrm{kHz}(\mathrm{r}=0.42, \mathrm{p}=0.01)$ (Figure 2(b)), respectively. In addition, THI score was significantly associated with ABG $(r=0.60, p<0.01)$ (Figure $2(c)$ ).

\subsection{Typical Case}

During the study period, $\mathrm{BC}$ hearing frequency of one patient among the $34 \mathrm{pa}-$ tients presenting tinnitus before surgery returned to their normal states after the therapy. The typical audiogram could be found in Figure 3.

\section{Discussion}

The etiological relationship between SNHL and Otitis Media (OM), consisting of Acute Otitis Media (AOM) [5] [9] [10], Chronic Otitis Media (COM) [6] [11] [12], Otitis Media with Anti-neutrophil Cytoplasmic Antibody (ANCA) [13], Eosinophilic Otitis Media (EOM) [14], cholesteatoma with labyrinthine fistula [15] and reflux-related otitis media [16], has been reported by numerous researchers. However, studies between SNHL and SOM are relatively rare. It was reported that the $\mathrm{BC}$ hearing loss could reach $25 \mathrm{~dB}$ or even higher level at any

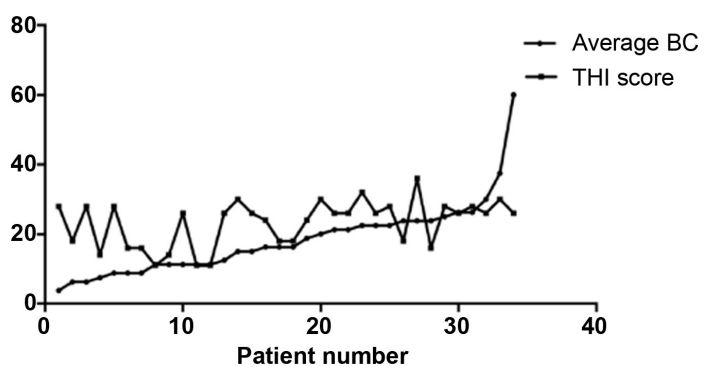

(a)

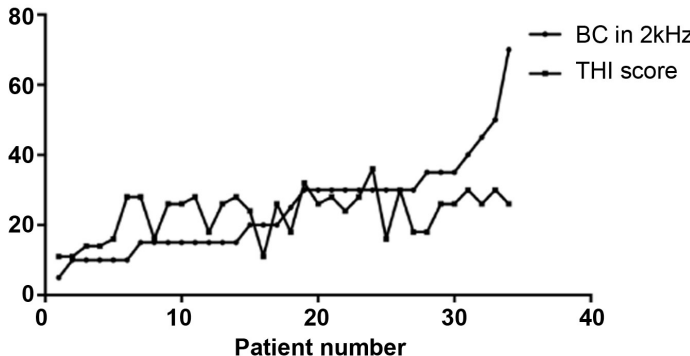

(b)

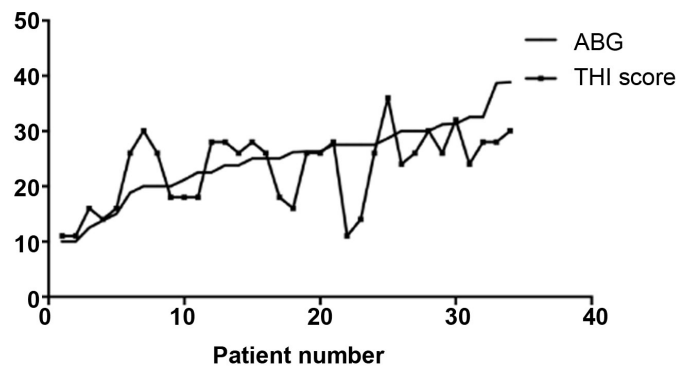

(c)

Figure 2. (a) Correction between Average BC hearing frequency and THI score; (b) Correction between BC frequency in $2 \mathrm{kHz}$ and THI score; (c) Correction between ABG and THI score. 


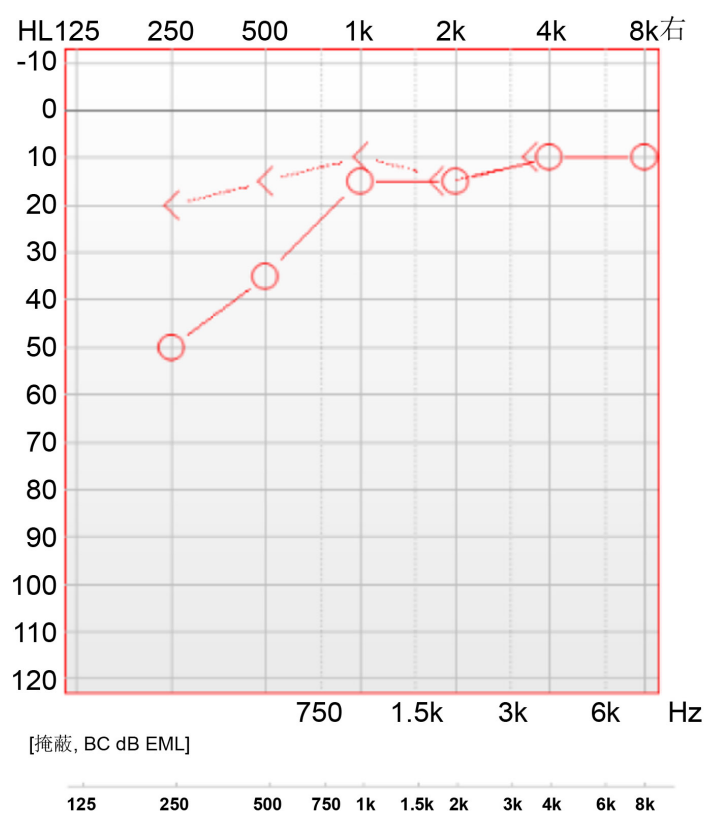

(a)

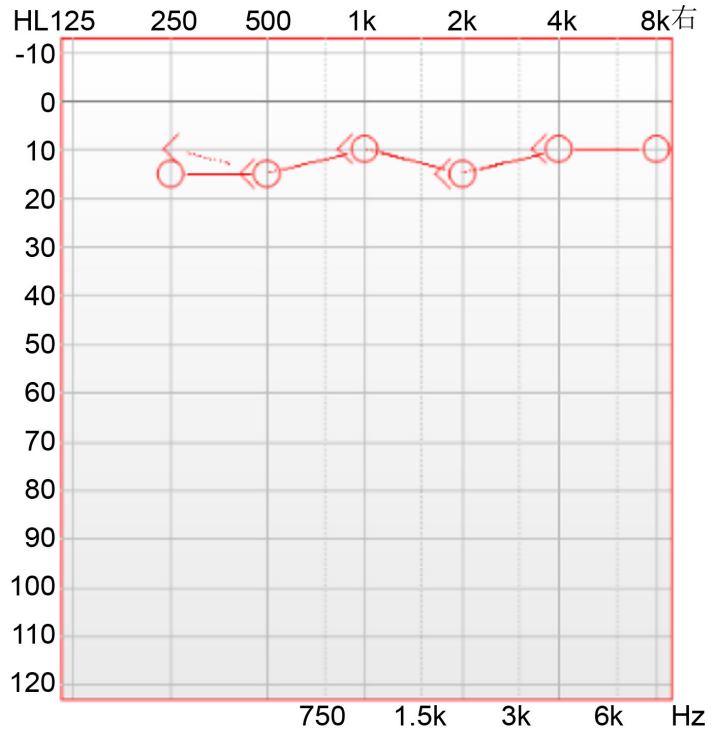

[掩蔽, $B C \mathrm{~dB} E M L]$

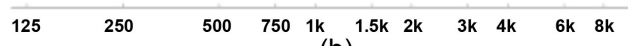

(b)

Figure 3. Audiogram of typical case (a) preoperatively; (b) postoperatively.

frequency from $0.25 \mathrm{kHz}$ to $4 \mathrm{kHz}$ for the $\mathrm{SOM}$ patients in comparison with normal cases. Personally, the shortage refers to that some obscure SNHL might be neglected.

All the patients enrolled were suffered from SOM totally confined to the single side and the comparison was taken between the affected side and non-affected side. Using this method, we excluded the presbyacusis, otosclerosis as well as some diseases leading to $\mathrm{BC}$ loss of both sides. Subsequently, our study indicates that SNHL were found in the affected sides of SOM cases, compared with their counterparts, which conformed to the result of Harada [4]. The inner and middle ear are connected mainly based on the round and oval windows. In addition, these membranes may allow several toxins in the middle ear to pass through, from which we may account for why SOM could result in SNHL.

Tinnitus can be defined as a sound perception in the absence of sound input external to the patient. Subjective tinnitus is considered as the most common type, which is audible only to the patient, without internal or external sound input [17]. The etiology of tinnitus is frequently multifactorial, yet hearing loss is the most risk factors in a lot of cases, [18]. Tinnitus remains as a common problem for patients with chronic otitis media, of which the incidence was reported as $43 \%$ [19]. However, tinnitus could also be a puzzle for patients with SOM, bringing about inner ear deficits to some extent. Our results demonstrated that the THI symptom, correlated with conductive hearing loss, was reduced but not eliminated after the operation, showing consistence with the result of Kim [19], indicating that tinnitus in SOM has partial correlation with the SNHL.

Briefly, simultaneous improvements of tinnitus symptom and SHHL verify the hypothesis that inner ear damages caused by SOM could be alleviated, therefore 
affording better qualities of life for SOM patients following myringotomy. Tinnitus could be relieved along with the improvements of conductive hearing loss, but it still remained for the SNHL remained.

\section{Conclusion}

To conclude, SOM got the damage on SNHL and tinnitus. Tinnitus shows correlation with conductive hearing loss and SNHL. In addition, proper and early therapeutic could release the deficits.

\section{Funding}

This work was supported by Xi'an Science and Technology Project [2017119SF/YX013(3)] to Ying Gao, and The K \& D Projects in Shaanxi [2017SF-182] to Ying Gao.

\section{Availability of Data and Materials}

The datasets used and/or analyzed during the current study are available from the corresponding author on reasonable request.

\section{Ethics Approval and Consent to Participate}

Ethics approval was obtained from The Second Affiliated Hospital of Xi'an Jiaotong University.

\section{Conflicts of Interest}

The authors declare that they have no competing interests.

\section{References}

[1] Atkinson, H., Wallis, S. and Coatesworth, A.P. (2015) Otitis Media with Effusion. Postgraduate Medicine, 127, 381-385. https://doi.org/10.1080/00325481.2015.1028317

[2] Rosenfeld, R.M., Shin, J.J., Schwartz, S.R., Coggins, R. and Gagnon, L. (2016) Clinical Practice Guideline: Otitis Media with Effusion Executive Summary (Update). Otolaryngology-Head and Neck Surgery, 154, S1-S41. https://doi.org/10.1177/0194599815624407

[3] Sone, M. (2017) Inner Ear Disturbances Related to Middle Ear Inflammation. Nagoya Journal of Medical Science, 79, 1-7.

[4] Harada, T., Yamasoba, T. and Yagi, M. (1992) Sensorineural Hearing Loss Associated with Otitis Media with Effusion. ORL Journal for Otorhinolaryngology and Its Related Specialties, 54, 61-65. https://doi.org/10.1159/000276263

[5] Park, J.H., Park, S.J., Kim, Y.H. and Park, M.H. (2014) Sensorineural Hearing Loss: A Complication of Acute Otitis Media in Adults. European Archives of Oto-Rhino-Laryngology, 271, 1879-1884. https://doi.org/10.1007/s00405-013-2675-x

[6] Kalaycik Ertugay, C., Kulekci, S., Naiboglu, B., Ertugay, O.C. and Kaya, K.S. (2015) Clinical Evaluation of Inner Ear Changes as Result of Chronic Otitis Media and Its Surgery. Kulak Burun Bogaz Ihtisas Dergisi, 25, 22-27. 
https://doi.org/10.5606/kbbihtisas.2015.36048

[7] Yoshida, H., Miyamoto, I. and Takahashi, H. (2010) Is Sensorineural Hearing Loss with Chronic Otitis Media Due to Infection Or Aging in Older Patients? Auris Nasus Larynx, 37, 402-406. https://doi.org/10.1016/j.anl.2010.01.001

[8] Geven, L.I., Koppl, C., de Kleine, E. and van Dijk, P. (2014) Plasticity in Tinnitus Patients: A Role for the Efferent Auditory System? Otology \& Neurotology, 35, 796-802. https://doi.org/10.1097/MAO.0000000000000307

[9] Newman, C.W., Jacobson, G.P. and Spitzer, J.B. (1996) Development of the Tinnitus Handicap Inventory. Archives Otolaryngology Head Neck Surgery, 122, 143-148. https://doi.org/10.1001/archotol.1996.01890140029007

[10] Jiang, S., Seale, T.W. and Gan, R.Z. (2016) Morphological Changes in the Round Window Membrane Associated with Haemophilus Influenzae-Induced Acute Otitis Media in the Chinchilla. International Journal of Pediatric Otorhinolaryngology, 88, 74-81. https://doi.org/10.1016/j.ijporl.2016.06.049

[11] Yang, C.J., Kim, T.S., Shim, B.S., Ahn, J.H. and Chung, J.W. (2014) Abnormal CT Findings Are Risk Factors for Otitis Media-Related Sensorineural Hearing Loss. Ear Hearing, 35, 375-378. https://doi.org/10.1097/AUD.0000000000000015

[12] Kaya, S., Tsuprun, V., Hizli, O., Schachern, P.A. and Paparella, M.M. (2016) Cochlear Changes in Serous Labyrinthitis Associated with Silent Otitis Media: A Human Temporal Bone Study. American Journal of Otolaryngology, 37, 83-88. https://doi.org/10.1016/j.amjoto.2015.10.002

[13] Yoshida, N. and Iino, Y. (2014) Pathogenesis and Diagnosis of Otitis Media with ANCA-Associated Vasculitis. Allergology International, 63, 523-532. https://doi.org/10.2332/allergolint.14-RAI-0774

[14] Matsubara, A., Nishizawa, H., Kurose, A., Nakagawa, T. and Takahata, J. (2014) An Experimental Study of Inner Ear Injury in an Animal Model of Eosinophilic Otitis Media. Acta Oto-Laryngologica, 134, 227-232. https://doi.org/10.3109/00016489.2013.859395

[15] Sone, M., Yoshida, T., Naganawa, S., Otake, H. and Kato, K. (2012) Comparison of Computed Tomography and Magnetic Resonance Imaging for Evaluation of Cholesteatoma with Labyrinthine Fistulae. Laryngoscope, 122, 1121-1125. https://doi.org/10.1002/lary.23204

[16] Develioglu, O.N., Yilmaz, M., Caglar, E., Topak, M. and Kulekci, M. (2013) Oto-Toxic Effect of Gastric Reflux. Journal of Craniofacial Surgery, 24, 640-644. https://doi.org/10.1097/SCS.0b013e31827c7dad

[17] Levine, R.A. and Oron, Y. (2015) Tinnitus. Handbook of Clinical Neurology, 129, 409-431. https://doi.org/10.1016/B978-0-444-62630-1.00023-8

[18] Baguley, D., McFerran, D. and Hall, D. (2013) Tinnitus. Lancet, 382, 1600-1607. https://doi.org/10.1016/S0140-6736(13)60142-7

[19] Kim, D.K., Park, S.N., Kim, M.J., Lee, S.Y. and Park, K.H. (2011) Tinnitus in Patients with Chronic Otitis Media before and after Middle Ear Surgery. European Archives of Oto-Rhino-Laryngology, 268, 1443-1448.

https://doi.org/10.1007/s00405-011-1519-9 